\title{
New Technologies in Radiation Oncology
}

\author{
W. Schlegel, T. Bortfeld, and A.-L. Grosu, eds.
}

New York, NY: Springer, 2006, 459 pages, \$269

New Technologies in Radiation Oncology provides an excellent overview of recent technologic developments in the field of radiation oncology. This book is intended for physicians and medical physicists who are working in radiation oncology and those just entering the field. It encompasses a broad range of new technologies in radiation therapy-from image acquisition and processing to treatment planning to therapy - and helps the reader grasp the basic ideas of each new technology. If the reader wants more information than the book provides, each chapter includes a wealth of references for learning about a particular subject in detail.

The book begins with a preface by the editors that includes a brief history of recent technologic milestones, including linear accelerators, CT/MRI-based 3-dimensional (3D) planning, stereotactic radiotherapy, 3D conformal radiation therapy with multileaf collimators, intensity-modulated radiotherapy, proton or ion therapy, and image-guided radiotherapy. The many new technologies that have emerged since the 1960s clearly demonstrate the rapid changes that have occurred in the field of radiation oncology and highlight the important contribution of this book in helping busy practicing physicians and physicists to stay up to date on new technologies.

One of the editors provides a review chapter covering the framework for the major sections of the book: the basics of 3D imaging, 3D imaging for radiotherapy, 3D treatment planning for conformal radiotherapy, new treatment techniques, and verification and quality assurance. This informative overview connects the technologies covered in the 5 major sections of the book. The basic steps in radiation therapy-immobilization, imaging, tumor localization, treatment planning, patient positioning, and treatment-are briefly discussed. This is a mustread chapter for those just entering the field.

The section on the basics of 3D imaging includes chapters on image reconstruction, segmentation, visualization, registration, and fusion, as well as data formats, networking, archiving, and telemedicine. The image reconstruction chapter is brief and could be reasonable for the radiation oncology community. The other chapters provide excellent overviews of the tools of image processing and data communication. Illustrations of images are abundant, and the

COPYRIGHT ( $) 2008$ by the Society of Nuclear Medicine, Inc. DOI: $10.2967 /$ jnumed.107.048827 reader will find the concepts of many image-processing techniques, as well as data communication protocols, presented in a relatively straightforward manner.

The section on 3D imaging for radiation therapy provides an excellent introduction to the applications of multislice CT, 4-dimensional CT, MRI and MR spectroscopy, PET and PET/CT, and ultrasonography. These imaging modalities are all of great importance to radiation therapy. An in-depth description of multislice CT and an introduction to conebeam CT are provided. The practice of using 4-dimensional CT to estimate tumor motion in the thorax, which has gained acceptance in many institutions, is covered in detail. Excellent discussions of tissue contrast with MRI and functional imaging of chemical composition with MR spectroscopy are also included. These chapters are written primarily from the perspective of radiation therapy, and they provide the reader with an understanding of how the images acquired from different imaging modalities are used in treatment planning. The organization and presentation of this section are excellent. As image-guided radiation therapy becomes more prevalent, the materials covered in this section will become more important to practicing physicians and physicists. The section would have benefited, however, from the inclusion of SPECT.

In the next main section of the book, 3D treatment planning for conformal radiation therapy, new concepts of biologic target volume defined by the images of PET, SPECT, or MR spectroscopy are introduced and examples of their applications to different anatomic sites are given. Different algorithms for dose calculation are discussed, and some insights are provided. In the chapter on optimization of treatment planning, the authors Bortfeld and Thieke provide a practical strategy for inverse planning and give a clinical example to demonstrate its applicability.

The fourth section, which explains the concepts behind almost all modern techniques currently in clinical use, is probably the most exciting section of the book. As treatment machines become more sophisticated and more tools become available to improve accuracy, clinical physicists and oncologists need sources of concise information on the new techniques available to evaluate what each entails.

The fifth, and last, section looks at quality assurance related to the use of modern radiation therapy techniques. As in previous sections of the book, the concepts are well 
explained, and references are provided for those interested in a more in-depth review of each quality assurance technique.

The practice of radiation oncology relies on the accuracy of information presented by both anatomic and functional images. It is not possible for anyone to understand every subject in detail, but this book provides an excellent treatment of each subject for practicing physicians and physicists in radiation therapy and also provides enough references for anyone interested in having more detailed knowledge.
Tinsu Pan Dershan Luo M.D. Anderson Cancer Center Houston, Texas 